\title{
Evaluación financiera del sistema de producción de papa (Solanum tuberosum L) en Oicatá, Boyacá
}

\section{Financial evaluation of the potato production system (Solanum tuberosum L.) in Oicatá, Boyacá}

Fecha de recepción: 27 de enero de 2015

Elmer Avendaño-Gómez ${ }^{1}$

Fecha de aprobación: 23 de abril de 2015

Wilson González-Santos ${ }^{2}$

\section{Resumen}

La factibilidad financiera es fundamental para tomar decisiones en un sistema productivo; en consecuencia, toda actividad agropecuaria debe hacer uso de dicha herramienta, a fin de obtener mayor comprensión de su ejercicio financiero y, a la vez, tener un soporte objetivo para determinar, entre otras cosas, si continuar con la actividad, diversificarla, combinarla o arrendar el espacio físico donde se realiza (1). El objetivo de este trabajo fue realizar una evaluación financiera en el sistema de producción de la papa Solanum tuberosum, variedad Diacol capiro, de manera participativa y con la inclusión de indicadores históricos, en función de horizontes de tiempo y tasa de oportunidad. La población objeto del estudio correspondió a 32 agricultores de papa inscritos en la Empresa Prestadora de Servicios Técnicos Agropecuarios (Epsagro) del municipio de Oicatá, departamento de Boyacá, para el año 2014, de los cuales se seleccionaron tres productores (grande, mediano y pequeño) para realizar la investigación. Los resultados indican que el diagnóstico rural participativo fue una herramienta de investigación adecuada para la compilación de la información, además de la existencia de diferencias en la estructura de costos de producción en los diferentes agricultores. En el municipio de Oicatá se encontró que existen fallas relevantes a nivel operativo y contable que deben ser corregidas.

Palabras clave: Diagnóstico rural participativo, factibilidad financiera, producción de papa en Boyacá.

\begin{abstract}
Financial feasibility is essential for decision making in a production system. Therefore all agricultural activity should use this tool to gain a greater understanding of its finantial exercise and also have an objective support to determine among other things whether to continue with the activity, diversify, combine it or lease the physical space where it is performed. The aim of this work is to conduct a financial

1 Profesional independiente (Tunja-Boyacá, Colombia).

2 Ph.D. (c) Universidad Pedagógica y Tecnológica de Colombia (Tunja-Boyacá, Colombia).wilson.gonzalez@uptc.edu.co.
\end{abstract}


evaluation in the production system of potato Solanum tuberosum variety Diacol capiro, in a participatory manner and with the inclusion of historical indicators, depending on time horizons and rate chance.

The study population corresponded to 32 potato farmers enrolled in the company providing agricultural technical services (Epsagro) Oicatá Township, Boyacá, 2014, of which 3 producers (large, medium and small) were selected for the research. The results indicate that the participatory rural diagnosis was a proper research tool for information, also there were differences in the structure of production cost in the different farmers. Besides in the municipality of Oicatá was found that there are flaws in relevant operational and accounting standards, that should be corrected.

Keywords: Financial feasibility, participatory rural diagnosis, potato production in Boyacá. 


\section{Introducción}

La papa (Solanum tuberosum) es el cultivo hortícola con mayor volumen de producción en el mundo, y el cuarto después de los cereales; se siembra en más de 95 países (2). El tubérculo es originario de los Andes suramericanos; en Colombia se concentra en paisajes y ecosistemas de montaña, entre los 2.000 y $3.500 \mathrm{msnm}$, pues alturas inferiores o superiores a dichas cotas se consideran marginales para el cultivo. La zona óptima de producción para las variedades Solanum tuberosum spp. andigena y Solanum phureja oscila entre los 2.500 y 3.000 msnm (3).

En Colombia, la papa (Solanum tuberosum L.) es uno de los cultivos más importantes del país. Los cuatro mayores productores son Cundinamarca $(39 \%)$, Boyacá $(27 \%)$, Nariño $(16 \%)$ y Antioquia (10\%), con 108.308 hectáreas, una producción de 1867.899 toneladas y un rendimiento promedio, en el 2010, de 17.2 t/ha (4). La producción del tubérculo en Boyacá está localizada en 82 municipios; en especial, en el municipio de Oicata es el cultivo transitorio de mayor importancia económica, y junto con los cereales, la arveja y el frijol, lideran la agricultura (5).

El análisis de factibilidad financiera es el elemento fundamental para la toma de decisiones en un sistema productivo. Toda actividad agropecuaria debe hacer uso de dicha herramienta, con el fin de obtener mayor comprensión de su ejercicio y tener un soporte objetivo para determinar si continuar con la actividad, diversificarla, combinarla o arrendar el espacio físico donde se realiza (1).

Una de las principales falencias del sistema de producción de la papa es la carencia de información de los distintos rubros que componen su proceso productivo, a partir de la participación activa de los actores implicados en su producción. La productividad colombiana en papa, al ser comparada con dos grandes potencias, como lo son China y los EE.UU., presenta algunas diferencias relacionadas con menores rendimientos

(6) y mayor precio de comercialización para los productos frescos (7).
A partir de un estudio con agricultores de papa en el municipio Oicatá, Boyacá, la presente investigación pretende contribuir a llenar los vacíos existentes, explicitados anteriormente. Así, el objetivo del trabajo es realizar una evaluación financiera en el sistema de producción de la papa Solanum tuberosum, variedad Diacol capiro, de manera participativa y con la inclusión de indicadores históricos, que -en función de horizontes de tiempo y tasa de oportunidad- tengan en cuenta la pérdida del dinero en el tiempo y evidencien el sesgo que se comete al evaluar financieramente cultivos transitorios con el uso exclusivamente de indicadores temporales.

\section{Materiales y métodos}

La metodología se construyó y ajustó partir de diversos estudios $(8,9,10)$. Previamente se precisan algunos conceptos sobre el DRP y el estudio de factibilidad.

\section{Diagnostico Rural Participativo}

El Diagnostico Rural Participativo (DRP) es un conjunto de técnicas y herramientas que permiten que las comunidades hagan su propio diagnóstico y comiencen a autogestionar su planificación y desarrollo (11). Algunas de las principales ventajas del DRP son la participación activa de planificadores y comunidad, quienes de manera multidisciplinar verifican y ajustan la información, así como la inclusión de la perspectiva de género en los procesos de caracterización rural.

\section{Estudio de factibilidad}

El estudio de factibilidad contiene los indicadores de evaluación financiera trabajados en la investigación, y se define como el análisis de una empresa para determinar si el negocio que se propone será bueno o malo. Según Hofstrand (12), los estudios de factibilidad se pueden utilizar de muchas maneras, pero se centran en empresas de negocio. Los agricultores y otras personas con una idea de negocio deben realizar un estudio de factibilidad para determinar la viabilidad de su idea antes de proceder. 


\section{Localización}

La investigación se llevó a cabo en el municipio de Oicatá, Boyacá; ubicado a los $5^{\circ} 35^{\prime} 40^{\prime \prime}$ de latitud norte y $73^{\circ} 18^{\prime} 29^{\prime \prime} \mathrm{O}$ de longitud oeste, respecto al meridiano de Greenwich, a una altura de 3.150 msnm (13); cuenta con un área total de $59 \mathrm{~km}^{2}$, entre los pisos térmicos frío y páramo, con temperaturas entre 10 y $14{ }^{\circ} \mathrm{C}$ y con precipitación entre 600 y $1.000 \mathrm{~mm}$ anuales.
La investigación se desarrolló desde el enfoque metodológico mixto, con componentes propios de la investigación cualitativa y cuantitativa. La población objeto del estudio correspondió a 32 agricultores de papa inscritos en la Empresa Prestadora de Servicios Técnicos Agropecuarios (EPSAGRO) del municipio de Oicatá, de los cuales se seleccionaron tres productores (grande, mediano y pequeño), para realizar la investigación. Los atributos que soportan esta muestra, de naturaleza dirigida (14), se notan en el Tabla I.

Tabla I. Atributos para determinación de las fincas.

\begin{tabular}{ll}
\hline \multicolumn{1}{c}{ ITEM } & CARACTERÍSTICA \\
\hline Tenencia de la tierra & Propia \\
\hline Ubicación de la finca & Oicatá, Boyacá \\
\hline Inscripción a asociación & Epsagro \\
\hline Área de cultivo & Pequeños $<3$ ha, mediano 3-10 ha, grande $>10$ ha \\
\hline Lugar de venta del producto & Tunja, Boyacá \\
\hline Variedad de papa & Diacol capiro \\
\hline Uso de suelo & Rotación papa pastos \\
\hline
\end{tabular}

\section{Recolección de la información}

El instrumento que permitió la operativización de las herramientas del DRP fue la entrevista semiestructurada, que se conformó en función de distintos componentes:

- Labores y prácticas de cultivo más representativas utilizadas

- Rendimiento luego de la estandarización de unidades
- Labores y prácticas utilizadas en control de plagas y enfermedades

Se realizó la evaluación financiera comparativa con indicadores temporales e históricos: Tasa Interna de Retorno (TIR) y Valor Presente Neto (VPN).

En la investigación, tanto los agricultores de papa como el investigador interactuaron de manera constante para la recolección acertada de la información. Por su parte, el papicultor demostró y se comportó como un sujeto activo, participando

3 El estudio tuvo en cuenta la superficie como criterio clasificatorio del tipo de agricultor. El Ministerio de Agricultura y Desarrollo Rural (15), por consenso, determino que los agricultores de papa se clasificarán de acuerdo con el número de hectáreas del cultivo que contenían sus predios. 
de las diferentes actividades realizadas a lo largo de la investigación. Enseguida se presentan los resultados de la investigación.

\section{Resultados y discusión}

\section{Experiencias en la recolección de la información}

La investigación hizo referencia a un enfoque local, en donde el productor de papa no solo es actor sujeto de la investigación, sino también constructor de ella. La participación como elemento fundamental concibe unos procesos construidos con la población, no solo como fuente de información, sino también como fuente de reflexión y de solución a su problemática. El enfoque metodológico que direccionó la investigación permitió que los participantes experimentaran el proceso de aprendizaje "aprender a aprender (16).

\section{Definición de los rubros y flujos de fondos (Cash Flow)}

La fijación de los rubros y sus unidades, a fin de normalizar la información, se realizó de acuerdo con la experiencia con los agricultores de papa (Tablas II y III).

Tabla II. Rubros en el agroecosistema papero. ${ }^{3}$

\begin{tabular}{|c|c|c|}
\hline ETAPA & LABOR & UNIDAD \\
\hline Adecuación de terreno & $\begin{array}{l}\text { Subsolar, arar } \\
\text { Encalada }\end{array}$ & $\begin{array}{l}\mathrm{H} \\
\text { Bultos }\end{array}$ \\
\hline Material de siembra & $\begin{array}{l}\text { Semilla } \\
\text { Quebrar latencia }\end{array}$ & $\begin{array}{l}\text { Bultos } \\
\mathrm{mm} / \mathrm{m}^{3}\end{array}$ \\
\hline Establecimiento de cultivo & $\begin{array}{l}\text { Fertilizante } \\
\text { Siembra } \\
\text { Preemergente herbicida } \\
\text { Aplicación pre emergente } \\
\text { Insecticida } \\
\text { Aplicación insecticida } \\
\text { Fungicida } \\
\text { Aplicación fungicida }\end{array}$ & $\begin{array}{l}\text { g } \\
\text { Jornal o contrato } \\
\text { I/h } \\
\text { Jornal } \\
\mathrm{k} / \mathrm{h} \text { o l/ha } \\
\text { Jornal } \\
\mathrm{k} / \mathrm{ha} \mathrm{o} \mathrm{I/ha} \\
\text { Jornal }\end{array}$ \\
\hline Aporcada & $\begin{array}{l}\text { Desyerbe y aporque } \\
\text { Fertilización } \\
\text { Aplicación herbicida }\end{array}$ & $\begin{array}{l}\text { Jornal o contrato } \\
\text { Jornal o contrato } \\
\text { Jornal o contrato }\end{array}$ \\
\hline Cosecha & Recolección & Jornal o contrato \\
\hline
\end{tabular}

4 Para este estudio se entiende como agroecosistema un ecosistema que es intervenido por el hombre a frecuentes modificaciones de sus componentes bióticos y abióticos. 
Tabla III. Medidas de uso común para los papicultores.

MEDIDAS DE USO COMÚN PARA LOS PAPICULTORES

\section{DESCRIPCIÓN}

\begin{tabular}{cl}
\hline Bolsa & Presentación de insumos en $500 \mathrm{~g} \mathrm{o} 1000 \mathrm{~g}$ \\
\hline Tarros & Insumos de $250 \mathrm{~mm}, 500 \mathrm{~mm}, 1000 \mathrm{~mm}$ \\
\hline Caneca & Capacidad de $200 \mathrm{I}$ \\
\hline Carga & 2 bultos de $50 \mathrm{k}$ \\
\hline Batidas & Mezcla de pesticidas en recipientes de 200 I \\
\hline Tubos & Desyerbe en surcos \\
\hline Riche & Papa con el diámetro más pequeño \\
\hline Bombadas & Fumigadora espalda 20 I \\
\hline Reabone & Segunda fertilización \\
\hline
\end{tabular}

\section{Indicadores de evaluación}

Para el cálculo del VPN fue necesario definir el valor de la Tasa de Interés de Oportunidad (TIO). Se utilizó para el trabajo la mejor tasa reportada por entidades bancarias y cooperativas financieras existentes en el municipio de Tunja, para enero de 2015, la cual fue del 3\% EA.

\section{Estructura de costos}

Si bien es cierto que existe la dificultad de tener uniformidad en la estructura de costos para el cultivo de la papa, en razón, entre otros aspectos, de las múltiples variedades cultivadas y de las condiciones edafoclimáticas, sociales y económicas propias de cada región (17), las Tablas IV, V y VI muestran las estructuras de costos logradas de manera participativa para los pequeños, medianos y grandes productores en el municipio de Oicatá.

Tabla IV. Estructura de costos por hectárea, pequeño productor.

\begin{tabular}{|l|r|c|}
\hline \multicolumn{1}{|c|}{ ACTIVIDADES } & \multicolumn{1}{c|}{ VALOR \$ } & $\%$ \\
\hline Arada & 114.000 & $2,61 \%$ \\
\hline Caballoneada & 114.000 & $2,61 \%$ \\
\hline TOTAL LABOREO CULTIVO & 228.000 & $5,22 \%$ \\
\hline Siembra y primera abonada & 100.000 & $2,29 \%$ \\
\hline Control de malezas, aporque y fertilización & 175.000 & $4,00 \%$ \\
\hline Control de plagas y enfermedades & 250.000 & $5,72 \%$ \\
\hline Recolección y empacada & 180.000 & $4,12 \%$ \\
\hline Transporte interno & 130.000 & $2,97 \%$ \\
\hline Transporte externo & 184.000 & $4,21 \%$ \\
\hline SUBTOTAL LABORES & 1.019 .000 & $23,31 \%$ \\
\hline Semillas & 420.000 & $9,61 \%$ \\
\hline Herbicidas & & \\
\hline
\end{tabular}




\begin{tabular}{|l|r|c|}
\hline \multicolumn{1}{|c|}{ ACTIVIDADES } & VALOR \$ & $\%$ \\
\hline Insecticida & 70.000 & $1,60 \%$ \\
\hline Otros & 32.000 & $0,73 \%$ \\
\hline Fungicidas & 144.000 & $3,29 \%$ \\
\hline Fertilizantes compuestos & 780.000 & $17,84 \%$ \\
\hline Fertilizantes foliares & 300.000 & $6,86 \%$ \\
\hline Otros & 315.000 & $7,21 \%$ \\
\hline Empaques & 113.800 & $2,60 \%$ \\
\hline SUBTOTAL INSUMOS & 2.174 .800 & $49,75 \%$ \\
\hline Administración & 600000 & $13,72 \%$ \\
\hline Arrendamiento & 350000 & $8,01 \%$ \\
\hline TOTAL OTROS COSTOS & 950000 & $21,73 \%$ \\
\hline TOTAL & 4.371 .800 & $100 \%$ \\
\hline
\end{tabular}

Tabla V. Estructura de costos por hectárea, mediano productor.

\begin{tabular}{|l|r|r|}
\hline \multicolumn{1}{|c|}{ ACTIVIDADES } & \multicolumn{1}{c|}{ VALOR $\$$} & \multicolumn{1}{c|}{$\%$} \\
\hline Arada & 150.000 & $2,04 \%$ \\
\hline Caballoneada & 150.000 & $2,04 \%$ \\
\hline Aplicación correctivos & 50.000 & $0,68 \%$ \\
\hline Riego & 180.000 & $2,45 \%$ \\
\hline TOTAL ÁREA CULTIVO & 530.000 & $7,22 \%$ \\
\hline Siembra y primera abonada & 150.000 & $2,04 \%$ \\
\hline Control de malezas, aporque y fertilización & 292.000 & $3,98 \%$ \\
\hline Control de plagas y enfermedades & 400.000 & $5,45 \%$ \\
\hline Recolección y empacada & 800.000 & $10,90 \%$ \\
\hline Transporte interno & 150.000 & $2,04 \%$ \\
\hline Transporte externo & 180.000 & $2,45 \%$ \\
\hline TOTAL LABORES & 1.972 .000 & $26,86 \%$ \\
\hline Semillas & 437.500 & $5,96 \%$ \\
\hline Herbicidas & 33.000 & $0,45 \%$ \\
\hline Insecticida & 180.000 & $2,45 \%$ \\
\hline Otros & 32.000 & $0,44 \%$ \\
\hline Fungicidas & 52.500 & $0,72 \%$ \\
\hline Otros & 162.000 & $2,21 \%$ \\
\hline Fertilizantes compuestos & 2.160 .000 & $29,42 \%$ \\
\hline Coadyudante & 51.000 & $0,69 \%$ \\
\hline
\end{tabular}




\begin{tabular}{|l|r|r|}
\hline \multicolumn{1}{|c|}{ ACTIVIDADES } & \multicolumn{1}{c|}{ VALOR \$ } & \multicolumn{1}{c|}{$\%$} \\
\hline Correctivos & 300.000 & $4,09 \%$ \\
\hline Empaques & 152.400 & $2,08 \%$ \\
\hline TOTAL INSUMOS & 3.560 .400 & $48,49 \%$ \\
\hline Administración & 780.000 & $10,62 \%$ \\
\hline Arrendamiento & 500000 & $6,81 \%$ \\
\hline TOTAL OTROS & 1.280 .000 & $17,43 \%$ \\
\hline TOTAL & 7.342 .400 & $100,00 \%$ \\
\hline
\end{tabular}

Tabla V. Estructura de costos por hectárea, gran productor.

\begin{tabular}{|l|c|c|}
\hline \multicolumn{1}{|c|}{ ACTIVIDADES } & VALOR $\$$ & $\%$ \\
\hline Análisis de suelo & 99.000 & $1,14 \%$ \\
\hline Arada & 75.000 & $0,86 \%$ \\
\hline Cabolloneada & 62.500 & $0,72 \%$ \\
\hline Aplicación correctivos & 25.000 & $0,29 \%$ \\
\hline TOTAL AREA CULTIVO & 261.500 & $3,01 \%$ \\
\hline Siembra y primera abonada & 140.000 & $1,61 \%$ \\
\hline Control de malezas, aporque y fertilizada & 260.000 & $3,00 \%$ \\
\hline Control de plagas y enfermedades & 375.000 & $4,32 \%$ \\
\hline Otros & 75.000 & $0,86 \%$ \\
\hline Recolección y empacada & 919.000 & $10,59 \%$ \\
\hline Transporte interno & 100.000 & $1,15 \%$ \\
\hline Transporte externo & 644000 & $7,42 \%$ \\
\hline TOTAL LABORES & 2.513 .000 & $28,97 \%$ \\
\hline Semillas & 1.152 .000 & $13,28 \%$ \\
\hline Herbicidas & 58.000 & $0,67 \%$ \\
\hline Insecticida & 252.000 & $2,91 \%$ \\
\hline Otros & 56.000 & $0,65 \%$ \\
\hline Fungicidas & 86.000 & $0,99 \%$ \\
\hline Otros & 224.000 & $2,58 \%$ \\
\hline Fertilizantes compuestos & 1.680 .000 & $19,37 \%$ \\
\hline Fertilizantes foliares & 150000 & $1,73 \%$ \\
\hline Coadyudante & 75.000 & $0,86 \%$ \\
\hline Correctivos & 480.000 & $5,53 \%$ \\
\hline Empaques & 187.000 & $2,16 \%$ \\
\hline TOTAL INSUMOS & 4.400 .000 & $50,72 \%$ \\
\hline Administración & 900000 & $10,38 \%$ \\
\hline Arrendamiento & 600000 & $6,92 \%$ \\
\hline TOTAL OTROS COSTOS & 1500000 & $17,29 \%$ \\
\hline TOTAL & 8.674 .500 & $100,00 \%$ \\
\hline
\end{tabular}


Las estructuras de costos permiten indicar las notables diferencias entre los productores de papa. En primera instancia cabe destacar el sesgo en que se puede incurrir al comparar porcentualmente los rubros, si se tienen en cuenta las enormes distancias entre los costos: el costo para el gran agricultor es el doble del pequeño agricultor oicatense. Si bien es cierto que el valor de los insumos se comporta como el de mayor peso para todos los casos, su distribución porcentual cambia en cada grupo. Un ejercicio en este sentido muestra que el peso porcentual de los fertilizantes varía entre un $18 \%$ y $21 \%$ (pequeño y gran agricultor) respecto a cerca de un $30 \%$ para el mediano agricultor. Esta última situación puede explicarse en razón al aumento de rubros en la medida que hay mayor capitalización: el uso de semilla certificada acarrea un valor significativo que (para el caso del gran agricultor) cambia las proporciones entre los demás rubros.

Los resultados contrastan con los indicadores temporales obtenidos en la investigación luego de realizar el flujo de caja libre. Se acepta que el VPN positivo en todos los casos permite inferir la bondad financiera del agroecosistema. No obstante, la rentabilidad cae dramáticamente entre nueve y quince puntos porcentuales, situación que evidencia el sesgo contenido en los estudios de valoración financiera de cultivos transitorios (Tablas VII y VIII)

Tabla VII. Valores de VPN y TIR para el agroecosistema papa.

\begin{tabular}{|c|c|c|}
\hline TIPO DE PRODUCTOR & VPN (3\% EA) \$ & TIR \% \\
\hline Grande & 1844505 & $7 \%$ \\
\hline Mediano & 998167 & $5 \%$ \\
\hline Pequeño & 752197 & $6 \%$ \\
\hline
\end{tabular}

Tabla VIII. Rentabilidad Diacol Capiro 2014.

\begin{tabular}{|l|l|l|l|}
\hline Ítem/Tipo de agricultor & Pequeño & Mediano & Grande \\
\hline Costos directos \$ & 3421 & 6062 & 7174 \\
\hline Costos indirectos \$ & 950 & 1280 & 1500 \\
\hline Costos totales \$ & 4371 & 7342 & 8674 \\
\hline Valor de la producción \$ & 5166 & 8400 & 10603 \\
\hline Ingreso neto \$ & 795 & 1058 & 1929 \\
\hline Rentabilidad \% & 18,188058 & 14,410242 & 22,238875 \\
\hline
\end{tabular}

Nótese que el valor de la rentabilidad para todos los casos es positivo. Es de anotar que la menor tasa se encuentra en el mediano productor. En este sentido, si bien es cierto el pequeño agricultor registra el menor valor de producción, tiene una relación favorable respecto a sus costos. Estudios realizados por González (18) indican que la permanencia de la cadena agroalimentaria de la papa puede explicarse por su cercanía con los condicionantes de la economía campesina, en especial con la maximización de la renta agrícola familiar, con el incremento del autoconsumo 
y la disminución de los factores externos a la producción.

De acuerdo con González (19), la disminución de los factores externos en el agroecosistema del cultivo de la papa se relaciona con insumos que provee el productor, dada la biodiversidad del arreglo espacial en su predio.

\section{Conclusiones}

Eldiagnóstico rural participativofueunaherramienta de investigación adecuada para la compilación de la información; no obstante, dicho proceso debe ser objeto de la normalización y homogeneización previa al análisis de la información.

El agroecosisetma del cultivo de la papa Diacol capiro en el municipio de Oicatá es sostenible financieramente para las tipologías de agricultores incluidos en el estudio.

Existen diferencias en la estructura de costos de producción en los diferentes agricultores. En especial, se destaca el ítem siembra, donde la proveniencia de la semilla es propia (pequeño productor), de zonas cercanas como Toca (mediano productor) y certificada (gran productor). Lo anterior se reporta en costos diferenciados para la tipología trabajada. Esta situación también se presenta en las bajas aplicaciones para el control químico que realizan los pequeños productores en comparación con los grandes agricultores de papa.

Después de realizar la recolección de información sobre el proceso productivo de las fincas en el municipio de Oicatá, se encontró que en estas existen fallas relevantes a nivel operativo y contable que deben ser corregidas para mejorar el desarrollo de las actividades.

\section{Referencias}

(1) Sánchez, J. Análisis de Rentabilidad de la empresa. Anal. C. (en línea) 2014 (fecha de acceso 1 de febrero de 2014). Disponible en http:// www.5campus.com/leccion/anarenta.
(2) Theodoracopoulos, M., Arias, S. y Ávila, H. Entrenamiento y desarrollo de Agricultores. 2008 (fecha de acceso 2 de abril de 2014). Disponible en http://www.mcahonduras. hn/documentos/PublicacionesEDA/Manuales $\% 20 \mathrm{de} \% 20$ produccion/EDA_Manual_Produccion_Papa_09_08.pdf.

(3) Herrera, C; Fierro H, y Moreno, J. D. Manejo integrado del cultivo de la papa. Produmedios; 2000.

(4) Villareal, H J. Situación actual de la cadena de la papa. Simposio de Actualización de la papa (Solanum tuberosum. L). Facultad de Ciencias Agropecuarias. Universidad Pedagógica y Tecnológica de Colombia; 2010.

(5) Alcaldía municipal de Oicata. Esquema de ordenamiento territorial. 1999. Capítulo IV, subsistema económico: 2-8.

(6) Kaiyun Xie. The status of organic potato production in Chinese. (en línea) 2008 (fecha de acceso 22 de diciembre de 2014). Disponible en http://www.fao.org/docrep/010/i0200e/ I0200E09.

(7) Bond J. Vegetables and pulses. (en línea) 2014 (fecha de acceso 28 de diciembre de 2014). Disponible en http://search.ers.usda.gov/search?affiliate $=$ ers\&query.

(8) Ochoa D. Evaluación financiera del sistema de producción de café especial en el municipio de Ocamonte. Tesis para optar por el título como ingeniero Agrónomo. Facultad de Ciencias Agropecuarias; Universidad Pedagógica y Tecnológica de Colombia, 2012.

(9) Perilla S. Análisis Financiero del Sistema de Producción Cafetalero en seis Municipios de la provincia de Vélez. Tesis para optar por el título como ingeniero Agrónomo. Facultad de Ciencias Agropecuarias; Universidad Pedagógica y Tecnológica de Colombia, 2012.

(10) Monroy G. Caracterización socioeconómica de la población rural de Tuta para su eventual 
participación en proyectos de producción de remolacha azucarera con destino a la industria de biocombustibles. Tesis para optar al título de ingeniero Agrónomo. Facultad de Ciencias Agropecuarias; Universidad Pedagógica y Tecnológica de Colombia, 2009.

(11) Verdejo, M. E. Diagnóstico rural participativo: una guía práctica: Centro Cultural Poveda; 2003.

(12) Hofstrand D. What is feasibility study? (en línea) 2009 (fecha de acceso 22 de diciembre de 2014). Disponible en http://www.extension.iastate.edu/agdm.

(13) Instituto Geográfico Agustín Codazzi. IGAC. Mapas municipios Boyacá. 2007.

(14) Ávila Baray, H.L. Introducción a la metodología de la investigación (en línea) 2006 (fecha de acceso 26 de diciembre de 2014) Disponible en http://www.eumed.net/libros/2006c/203/.

(15) Ministerio de Agricultura y Desarrollo Rural. Observatorio Agrocadenas Colombia. (en lí- nea) 2005 (fecha de acceso 22 de diciembre de 2014). Disponible en http://www.agronet. gov.co/www/docs_agronet/2005112163731.

(16) Balcázar, F. Investigación acción participativa (IAP): aspectos conceptuales y dificultades de implementación. Fundam. humanid. 2003; 1(7): 59-77.

(17) Espinal, C. y Martínez, F. La competitividad de las cadenas agroproductivas en Colombia: IICA. Ministerio de Agricultura y Desarrollo Rural; 2005.

(18) González, W. La permanencia de la cadena agroalimentaria de la papa en Colombia: una interpretación desde la economía campesina: Tercer Congreso de Horticultura: Sociedad Colombiana de Ciencias Hortícolas; 2009.

(19) González, W. 2009. Educación y entorno en la ingeniería agronómica: un encuentro entre la academia y su contexto: Universidad Pedagógica y Tecnológica de Colombia; 2009. 